\title{
Nanoencapsulation of Human Adipose Mesenchymal Stem Cells: Experimental Factors Role to Successfully Preserve Viability and Functionality of Cells
}

\author{
Daniel Hachim, Jaime Melendez, Roberto Ebensperger \\ Laboratorio de Terapia Celular y Medicina Regenerativa, Departamento de Farmacia, Facultad de Química, \\ Pontificia Universidad Católica de Chile, Santiago, Chile \\ Email: rebenspe@uc.cl
}

Received November 30, 2012; revised January 5, 2013; accepted January 17, 2013

\begin{abstract}
Cell nanoencapsulation is a novel delivery system based on a self-assembly technique mediated by electrostatic interactions called Layer-by-Layer (LbL) deposition, without an increase in volume implant because of the nanometric thickness of its layers. LbL coats the entire surface of individual cells, providing mechanical resistance to cells against manipulation and storage conditions prior to implantation in the patient. In this work, single-cell nanocapsule formation using human adipose-derived mesenchymal stem cells (ADSC) given their potentiality in regenerative medicine was assessed by fluorescence microscopy and Zeta potential assays. Both methodologies were conclusive in showing layerby-layer nanocapsule formation of every single ADSC. Significant differences in terms of viability and cell functionality preservation were observed depending on the polycation used. Using a combination of fluorescence microscopy and fluorimetric assays, we found that cell survival after nanocapsulation was only efficient when chitosan was added to cells. These results were consistent with other cell types used in this study. Other polycations such as poly(allylamine hydrochloride) (PAH), poly(diallyldimethylammonium chloride) (PDADMAC) and poly-L-lysine (PLL) markedly decreased cell viability $(22 \%, 11 \%$ and $15 \%$, respectively). In addition, the use of potassium-enriched saline solutions, such as Hanks and Ringer's solution, during the nanoencapsulation process on ADSCs was harmful on cell viability compared to standard media ( $36 \%$ vs $79 \%$, respectively). The addition of a mixture of polyanions such as hyaluronic acid and chondroitin sulfate did not affect cell viability ( $79 \%$ and $81 \%)$. The combination of chitosan/hyaluronic acid and chondroitin sulfate was also effective in preserving the cell functionality of ADSCs, including the proliferation and differentiation of these cells as assessed by MTT assay and microscopy, respectively. Taken together, these results indicate that ADSCs can be successfully nanoencapsulated using a first layer of chitosan and a second layer of a combination of hyaluronic acid and chondroitin sulfate with a standard potassium concentration in the culture medium.
\end{abstract}

Keywords: Mesenchymal Stem Cells; Nanoencapsulation; Polyelectrolytes; Chitosan

\section{Introduction}

The emerging use of stem cells allows treatment of multiple diseases where there is a loss of tissue or cell functionality [1]. Once the stem cells are implanted, they are capable of differentiating into multiple cell subtypes (depending on the implantation site) and secreting growth and tissue regeneration factors [1]. In addition to their intrinsic hypoimmunogenicity and immunomodulatory capability [2,3], their implantation is feasible due to allogeneic sources, and they have important implications in autoimmune diseases therapies [3].

Human mesenchymal stem cells (hMSC) are a type of adult stem cell; they originate from a mesenchymal embryonic lineage and have the potential to generate under determined conditions, different cell subtypes that are committed to developing into osteoblasts, chondrocytes, and adipocytes, among others $[4,5]$. Stem cells can be found in most tissues; adipocyte-derived stem cells (ADSC), however, are mainly derived from liposuctions which is generally considered as a biological waste [6].

To improve on and preserve ADSC features, procedures to create a scaffold that facilitates cell implantation have been developed, offering shell and protection against external conditions such as manipulation, transport and storage prior to implantation into the patient and thus preserving cell viability and functionality without a dramatic increase in implant volume. Nanoencapsulation offers all of these advantages [7-9]. By definition, nanocapsules are a type of conformational coating with nanometric thickness that individually encapsulates cells 
[7-9]. Both features represent advantages over other types of capsules used as scaffolds [10] (e.g., micro- or macrocapsules) in other systems. Cell coating using nanocapsules does not impair the free diffusion of nutrients, waste and bioactive molecules, which are necessary for cells. On the other hand, individually encapsulated cells prevent the formation of necrotic cores, which avoids the initiation of the immunological response to the implant, as the small pore size in every nanocapsule provides immunoisolation by blocking immunoglobulins' access to the cells $[8,9]$. Typical scaffolds encapsulate multiple cells per capsule, similar to microcapsules, whereas the diffusion of nutrients in the central zones of microcapsules is deficient [7-9,11].

Cell nanoencapsulation is based on the technique called Layer-by-Layer (LbL) deposition. LbL is formed by polymeric layers or film depositions that are mediated by alternating opposite electrostatic interactions on a charged template [12]. This process is feasible because cells are negatively charged on the surface membrane. Although this technique has been recognized as an easy and inexpensive method and successfully applied in prokaryotes, attempts to encapsulate mammalian cells have been inconsistent due to deficiencies in cell viability $[8,9$, 12-17]. Krol et al. have successfully nanoencapsulated yeast and determined its functionality in several publications [8]. Additionally, they have encapsulated pancreatic islets to mainly provide immunoisolation of these islets once implanted [9]; however; there are two major concerns in their findings. First, they evaluated cell viability through trypan blue staining only. As we know, central cores of pancreatic islet are highly packed/dense, so trypan blue staining could not be entering adequately. Although pancreatic islet could survive after nanoencapsulation, there are a high number of cells inside which are not in direct contact with polyelectrolyte films. Additionally, most of the cells in contact with polyelectrolite layers seem to be dead as revealed by trypan blue. In any case, this is not representative of a successfully single mammalian cell nanoencapsulation procedure. Second, antibody infiltration assays do not reflect any hypoimmunological response; they should perform a proliferation assay to corroborate such immunoisolation effect of nanocapsulation.

Veerabadran et al. have nanoencapsulated (PLL/HA) mouse mesenchymal stem cells [16], in which films created inside nanocapsules were well characterized; however, there is a lack of consistency in corroborating cell survival after this process.

Despite variable outcomes between different experimental conditions, we suggest that the effect of polycations and components of saline solutions are keys to sustain cell viability. Thus, we focused mainly on the most common polycations used in previous studies, which describe satisfactory outcomes [7-9,12-17]. In particular, in a recent study using different polycations to nanoencapsulate MELN cells, Germain and colleagues [17] showed that PDADMAC (poly-diallyldimethylamine chloride) remarkably resulted in a higher cell viability compared to PLL (poly-L-lysine), PAH (poly-allylamine), PS (Protamine Sulfate), PEI (Poly(ethylene imine)) and PPP (Poly(phosphoric acid)). Furthermore, they concluded that the use of potassium-enriched saline solutions such as Ringer's/ $\mathrm{KCl}$ and the standard requirements of calcium and magnesium ostensibly increased cell viability.

The present work describes the development of a successful nanoencapsulation procedure for ADSC based on Layer-by-Layer deposition and its use and applications in preserving both cell viability and functionality. Natural biopolymers that constitute important structural components of the extracellular matrix [18-21], such as chitosan, hyaluronic acid and chondroitin sulfate, were also assayed in this study. Furthermore, the choice of the best saline solution to preserve these cells during nanoencapsulation was also evaluated. The cell viability of different polyelectrolyte (polycations and polyanions) depositions and saline solutions was determined after nanoencapsulation. The evaluation of cell functionality was based on cell adhesion, proliferation (growth) and differentiation [4]. We found that the cell viability and functionality of ADSCs are not totally dependent on the polycationic layer; they are also dependent on the chemical composition of saline solutions due to their regulation of ionic strength and $\mathrm{pH}$ control.

\section{Materials \& Methods}

\subsection{Reagents}

Chitosan low mass weight, PAH; poly(allylamine hydrochloride) $15 \mathrm{kDa}$, PDADMAC; poly (diallyldimethylammonium chloride) very low molecular weight, PSS; poly(sodium 4-styrenesulfonate) $70 \mathrm{KDa}$, saponin, dexamethasone, 3-isobutyl-1-methylxanthine (IBMX), insulin (porcine), indomethacin, glycerol 2-phosphate, ascorbic acid 2-phosphate, Oil red O, Alizarin red S and poly-L-lysine were purchased from Sigma Aldrich. Hyaluronic acid was purchased from US Biological. Chondroitin sulfate from bovine trachea and hyaluronic acidFITC labeled from bovine trachea, DAPI and MTT viability assay kit were purchased from Calbiochem. Live/ Dead viability kit for mammalian cells was purchased from Invitrogen. Ringer's solution used in this study consisted of $10 \mathrm{mM}$ HEPES, $147 \mathrm{mM} \mathrm{NaCl}, 2 \mathrm{mM}$ $\mathrm{CaCl}_{2}, 1 \mathrm{mM} \mathrm{MgCl}, 5 \mathrm{mM}$ glucose, and $2 \mathrm{mM}$ sodium bicarbonate and was adjusted to $\mathrm{pH}$ 7.4. Ringer's $/ \mathrm{KCl}$ used in this study consisted of $147 \mathrm{mM}$ of $\mathrm{KCl}$ instead of $\mathrm{NaCl}$. 


\subsection{ADSC Isolation and Culture}

Abdominal fat tissue from lipoaspirates was digested with $0.1 \%$ collagenase $\mathrm{I}$ in DMEM $+2 \%$ BSA for 30 minutes at $37^{\circ} \mathrm{C}$ while continuously shaking. Fat was discarded after centrifugation at $200 \times \mathrm{g}$ for 5 minutes, and the cell pellet was washed twice with PBS. Cell strainers $(100 \mu \mathrm{m})$ were used to remove aggregations. The cell pellet was incubated in a solution of $\mathrm{NH}_{4} \mathrm{Cl}$ at $37^{\circ} \mathrm{C}$ for 10 minutes to eliminate erythrocytes. The cells were centrifuged $(200 \times \mathrm{g}, 5$ minutes $)$ and plated in culture bottles with DMEM $+10 \%$ FBS [22]. ADSCs were phenotypically characterized following the mesenchymal stem cell minimal criteria [4] by the presence of surface antigens, such as CD73, CD90 and CD105, and the absence of CD14, CD19, CD34, CD45 and HLA-DR. Cell functionality was also evaluated based on their adhesion to plastic plates, cell proliferation and ability to differentiate into adipocytes and osteoblasts.

\subsection{Nanoencapsulation of ADSCs}

A suspension of $1.2 \times 10^{6}$ ADSCs was washed with HBSS and centrifuged $(200 \times \mathrm{g})$ to discard the culture medium (DMEM $+10 \%$ FBS). The resulting cell pellet was resuspended in $0.5 \mathrm{~mL}$ of chitosan $(0.5 \mathrm{mg} / \mathrm{mL}$ in HBSS; pH 6.5), PAH, PDADMAC or PLL (1 mg/mL each; $\mathrm{pH} 7.4$ in HBSS) and incubated for 10 minutes at room temperature. The cells were washed twice using HBSS and collected by centrifugation to discard the non-adsorbed polyelectrolytes. For the chitosan-treated cells only, the resulting cell pellet was resuspended in a mixture of hyaluronic acid:chondroitin sulfate $(1 \mathrm{mg} / \mathrm{mL}$ 1:1 in HBSS; $\mathrm{pH} 7.4$ ), incubated for 10 minutes at room temperature and then washed twice with HBSS. To visualize the presence of nanoencapsulated ADSCs under a fluorescence microscope, FITC-labeled hyaluronic acid was used. The cell nuclei were co-stained with DAPI.

\subsection{Zeta Potential Measurements}

First, $4 \times 10^{7}$ Hela cells were suspended in $20 \mathrm{~mL}$ of PBS (control, no polyelectrolyte layer). In addition, a second sample of $4 \times 10^{7}$ Hela cells were covered with the first polycationic layer of Chitosan by the addition of $10 \mathrm{~mL}$ of chitosan $(0.5 \mathrm{mg} / \mathrm{mL}$ in HBSS; $\mathrm{pH} 6.5)$ and incubated for 10 minutes. Next, the cells were washed twice and resuspended in $20 \mathrm{~mL}$ of PBS (one polycationic layer). Finally, a third sample of $4 \times 10^{7}$ Hela cells were covered with a chitosan layer, as previously described, followed by a second layer of a mixture of hyaluronic acid:chon-

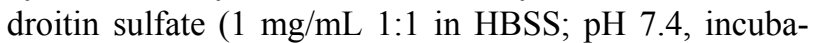
tion 10 minutes), then washed twice and resuspended in $20 \mathrm{~mL}$ of PBS (two layers, outer polyanionic layer). The zeta potential was measured on these 3 different cell samples as a mean of 10 readings using a Zeta Meter
System 3.0, Zeta-Meter, Inc. (Figure 1).

\subsection{Cell Viability Assessment after Polymeric Depositions}

A suspension of $1.2 \times 10^{6}$ ADSCs was washed with HBSS and centrifuged $(200 \times \mathrm{g})$ to discard the culture medium (DMEM). The resulting pellet was resuspended in $0.5 \mathrm{~mL}$ of chitosan solution $(0.5 \mathrm{mg} / \mathrm{mL} \mathrm{pH} 6.5$ in HBSS), PAH, PDADMAC or PLL (1 mg/mL each; $\mathrm{pH}$ 7.4 in HBSS). Next, the cells were incubated for $10 \mathrm{~min}$ utes at room temperature, washed with HBSS and centrifuged to eliminate any unabsorbed polycations. For the chitosan-treated cells only, the cell pellet was resuspended in $0.5 \mathrm{~mL}$ of a mixture of hyaluronic acid:chondroitin sulfate $(1 \mathrm{mg} / \mathrm{mL} 1: 1 ; \mathrm{pH} 7.4$ in HBSS $)$ and incubated for 10 minutes at room temperature. Next, the cells were washed twice with HBSS. Non-encapsulated cells (control) were obtained using the same procedure but in the absence of a polyelectrolyte solution.

\subsection{Cell Viability Assessment of Saline Solutions Effects on Cell Nanoencapsulation}

A suspension of $1.2 \times 10^{6}$ ADSCs was washed either with HBSS, Ringer's solution or their respective potassium supplemented solution, $\mathrm{HBSS} / \mathrm{KCl}\left(147 \mathrm{mM} \mathrm{K}^{+}\right)$or Ringer's $/ \mathrm{KCl}\left(147 \mathrm{mM} \mathrm{K}^{+}\right)$, and the cells were centrifuged $(200 \times \mathrm{g})$ to discard the culture medium. The resulting pellet was resuspended in $0.5 \mathrm{~mL}$ of chitosan solution dissolved in each of the solutions mentioned above $(0.5 \mathrm{mg} / \mathrm{mL}, \mathrm{pH} 6.5)$. Next, cells were incubated for 10 minutes at room temperature, washed with the corresponding saline solution and centrifuged to eliminate any unabsorbed polycations.

\subsection{Cell Viability Measurements}

Cell viability was performed using a Live/Dead assay kit

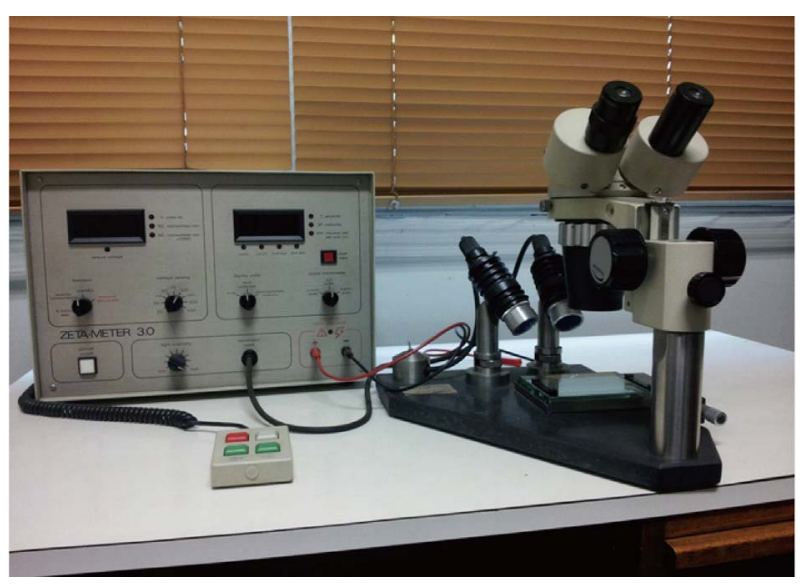

Figure 1. Picture ofthe zeta meter system 3.0 of zeta-meter, Inc. 
according to the manufacturer's instructions. Cells were plated $1 \times 10^{5}$ cells per well in DMEM in a 96-well plate after chitosan layer deposition. Next, a mixture of calcein-AM and ethidium homodimer was added and incubated for 40 minutes at room temperature. The cell viability was then measured on a FLX800 fluorimeter (Biotek). The percentage of dead cells was obtained from a ratio of dead cells in one sample, and the total number of dead cells was obtained in another sample by treatment with a $0.1 \%$ saponin solution. Fluorescent calcein (green, live cells) and ethidium homodimer (red, dead cells) were visualized under a fluorescence microscope.

\subsection{Cell Proliferation Assessment by MTT Quantification Assays}

To obtain synchronized ADSCs, cells were starved for 12 $\mathrm{h}$ and then nanoencapsulated. In addition, $3 \times 10^{4}$ cells were plated in a 96-well plate with DMEM plus $10 \%$ FBS. The cells were refed every two days with fresh culture medium. An MTT proliferation assay was performed at $0,2,4,6,8$ and 10 days after plating. Briefly, $10 \mu \mathrm{L}$ of MTT $(5 \mathrm{mg} / \mathrm{mL})$ was added to the cultured cells in a final volume of $110 \mu \mathrm{L}$ of culture medium (without Phenol Red) and incubated for an additional 3 hours at $37^{\circ} \mathrm{C}$ and $5 \% \mathrm{CO}_{2}$. Next, cells were treated with $100 \mu \mathrm{L}$ of a solution of acid isopropanol $\left(\mathrm{H}_{2} \mathrm{SO}_{4} 4 \mathrm{~N}\right.$ : Isopropanol $=1: 4)$ to determine the rate of cell proliferation by measuring the absorbance of formazan dye generated at 550 $\mathrm{nm}$. The same mixture in the absence of cells was used as a negative control. The same procedure was also used for non-nanoencapsulated cells.

\subsection{Cell Differentiation into Adipocytes and Osteoblasts}

For these sets of experiments, $2 \times 10^{5}$ nanoencapsulated ADSCs were cultured in DMEM $+10 \%$ FBS. For adipocyte differentiation, regular media was supplemented with dexamethasone $1 \mu \mathrm{M}$, IBMX $0.5 \mathrm{mM}$, insulin 10 $\mu \mathrm{g} / \mathrm{mL}$, and indomethacin $100 \mu \mathrm{M}$. The cells were refed every two days. After 21 days, cells were fixed with $10 \%$ formalin, washed and stained with a solution of Oil Red $\mathrm{O}: \mathrm{H}_{2} \mathrm{O}(3: 2)$ to reveal the presence of lipid drops [22] under the microscope. In the case of osteoblast differentiation, nanoencapsulated cells were treated with dexamethasone $100 \mathrm{nM}, \beta$-glycerophosphate $10 \mathrm{mM}$, and ascorbic acid 2-phosphate $50 \mu \mathrm{g} / \mathrm{mL}$. After 28 days, the cells were fixed with $10 \%$ formalin and stained with $1 \%$ Alizarin Red S for $1 \mathrm{~h}$ to reveal the presence of calcium phosphate deposits [22]. Non-nanoencapsulated cells were used as a control.

\section{Results and Discussion}

Stem cells are characterized by their capacity for self renewal and their ability to differentiate into specific cell types under the influence of their microenvironment. Cell encapsulation is a promising approach for long-term delivery of therapeutic agents, including cells. Mesenchymal stem cells (MSC), however, potentially may serve as a promising platform for cell-based encapsulation. They are known to be hypoimmunogenic and can be genetically modified to express a variety of therapeutic factors. A major advantage of stem cells is that they can be expanded ex vivo and transplanted. Ex vivo differentiation into specific cell types, or genetic modification to impart desired characteristics, enhances the therapeutic potential of stem cells. A variety of cellular deficiencies and dysfunctions such as diabetes, Alzheimer's disease, cancer, liver failure can be addressed with encapsulated cell therapy [23]. In this regard, besides achievements of human MSC microencapsulation described by Goren et al. [24], multilayer nanoencapsulation described by Bahiji et al. [25] and the protection against physical stress by layer-by layer cell coating described by Matsuzawa et al. [26], we have described the first successfully single cell nanoencapsulation of human adipose derived mesenchymal stem cells identifying an appropriate combination of polyelectrolytes that keep cell viability and functionality of the MSC for long-term cellular therapy application.

\subsection{Nanoencapsulation of ADSCs}

First, we conducted experiments to nanoencapsulate ADSCs. To demonstrate single-cell nanoencapsule formation, we used a FITC-labeled hyaluronic acid in the second layer after a deposition of a first layer of chitosan. The samples were evaluated by fluorescence microscopy after co-staining with DAPI (Figure 2). This image clearly shows the formation of a defined, strong and bright green fluorescent layer surrounding every single and isolated ADSC, thus demonstrating that nanoencapsulation occurred successfully in these cells, as revealed by DAPI staining. Evidence of single-cell nanoencapsulation of ADSCs with the most common polycations used for nanoencapsulation, such as PAH, PDADMAC and PLL, exhibited similar results, while the deposition of only polyanionic FITC-labeled hyaluronic acid without a previous polycationic layer did not show any evidence of nanoencapsulation (data not shown).

\subsection{Zeta Potential Measurements}

Additional assessments of polymeric layer depositions using zeta potential measures were performed and showed evidence of nanocapsule formation in two steps. Initially, the cells had a resting zeta potential of $-51 \mathrm{mV}$. After the first layer of chitosan (polycation), the zeta potential changed to $+36 \mathrm{mV}$. Finally, the zeta potential changed again to a negative value of $-108 \mathrm{mV}$ after the 


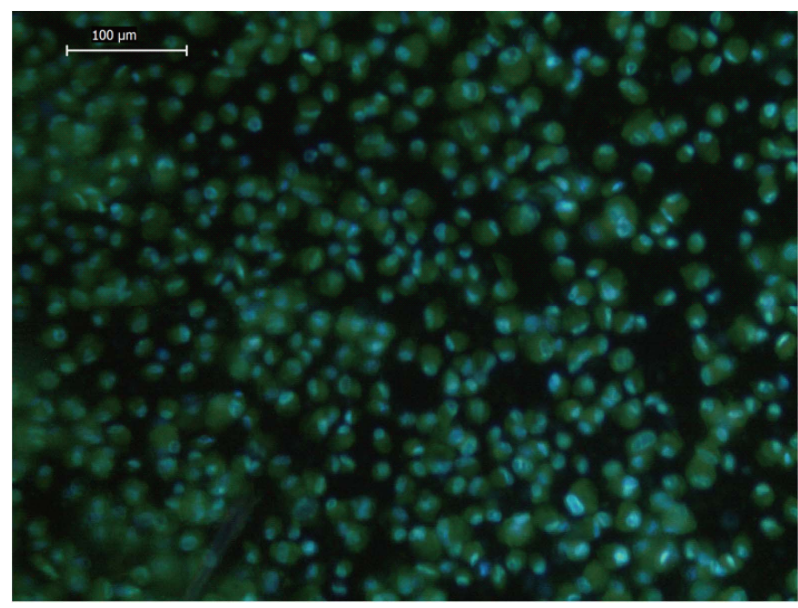

Figure 2. Nanoencapsulated adiposetissue derived stemcells (ADSC). The merged image shows nanoencapsulated ADSCs with chitosan as a first layer and HA-FITC:CS 1:1 as a second layer by fluorescence microscopy. Nuclei are represented in blue (DAPI) and FITC-labeled HA-polymer deposited on the cell surface in green. Magnification $200 \times$. This image is representative of at least 3 independent experiments.

deposition of a second layer of hyaluronic acid/chondroitin sulfate (polyanions). As shown in Figure 3, the cell has a negative zeta potential because its membrane surface is negatively charged; however, this charge was converted into a positive zeta potential after the deposition of a polycationic chitosan, which forms a positively charged film on the cell surface. Finally, a negative zeta potential was obtained because of the subsequent formation of a negatively charged polyanionic film of hyaluronic acid/chondroitin sulfate.

\subsection{Cell Viability of Nanoencapsulated ADSCs and the Effects of Polycationic Deposition}

To determine cell viability on nanoencapsulated ADSCs, the percentage of living cells was assayed by using a Live/Dead assay kit for mammalian cells. This kit is based on the use of two fluorophores: calcein-AM, which is not fluorescent when it is acetylated (thus, living cells incorporate and deacetylate it, generating a green fluorescent calcein), and ethidium, a homodimer that only enters dead cells with damaged cell membranes, conjugates to DNA and results in a red fluorescence.

First, nanocapsules were made of different polycations (chitosan, PAH, PDADMAC and PLL, the most common polycations used in nanoencapsulation) on ADSCs, and the effects of polycations on cell viability was evaluated (Figures 4(A) and (B)). Furthermore, to compare cell variability, we evaluated the effects of polycations on adherent Hela and non-adherent HL-60 cell lines. Chitosan did not show differences in cell viability when compared to non-encapsulated control cells ( $79 \%$ vs. $86 \%$ ).

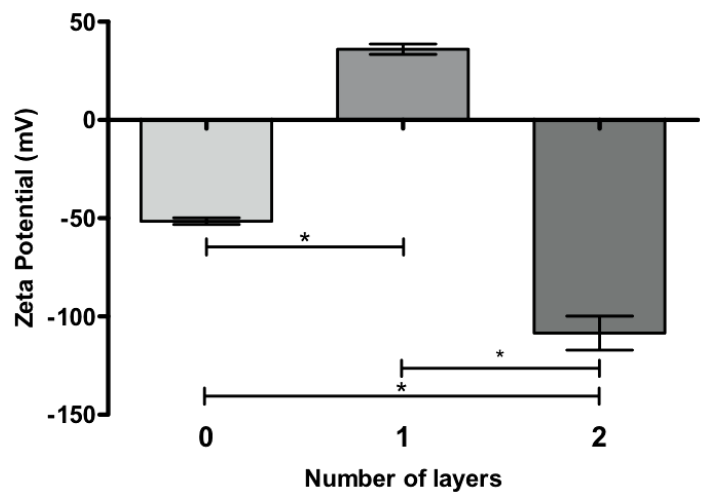

Figure 3. Determination of zeta potential. The graph represents the surface charge for uncoated Hela (0), chitos ancovered Hela (1) and Hela covered with a first layer of chitosan and a second layer of HA/CS 1:1 (2). The bars on the graph represent the mean of 10 Zeta potential measurements $+/-$ SEM. (*) p-value of less than 0.01 was statistically significant.

However, the addition of the polycations PAH, PDADMAC and PLL dramatically reduced the cell viability of ADSCs $(22 \%, 11 \%$ and 15\%, respectively; see Figure 4(A) and images on Figure 4(B)). These results were similar to other nanoencapsulated cell types such as Hela and HL-60s (Figure 4(A)), which exhibit a lower cell survival for PAH, PDADMAC and PLL and a high cell viability for controls and chitosan-covered cells.

It is remarkable how the addition of polycations can affect cell viability. This result could be explained if we consider that a negative resting potential of the cell membrane is needed to perform normal functions through the plasma membrane. Thus, after the first polycationic layer deposition, the membrane potential is altered, and some cell functions could be affected, resulting in reduced cell viability. A second polyanionic layer deposition should restore the membrane potential to a negative one by charge neutralization and maintain proper cell viability.

PDADMAC is a strong polyelectrolyte. It has a quaternary ammonium group, no $\mathrm{pH}$ and is ionic strengthdependent [27]. Thus, it is $100 \%$ positively charged, which largely alters the membrane potential, and may have led to mostly dead cells. Similar findings were found with PAH and PLL because they are weak polyelectrolytes $[12,28]$ and their amino groups have a pKa of approximately 9 . This indicates that their radical groups are almost fully charged at physiological $\mathrm{pH}$, resulting in a positive charge that greatly alters the membrane potential. Chitosan is a weak polyelectrolyte, and its amino groups have a pKa of 6.5 . They are $50 \%$ ionized at deposition with a $\mathrm{pH}$ of 6.5 [18], and thus, the charge density at the moment of deposition is lower, which does not affect cell viability. These observations give us some indication that $\mathrm{pH}$ control during polyelectrolyte deposition is a key factor in obtaining lower density charges. 


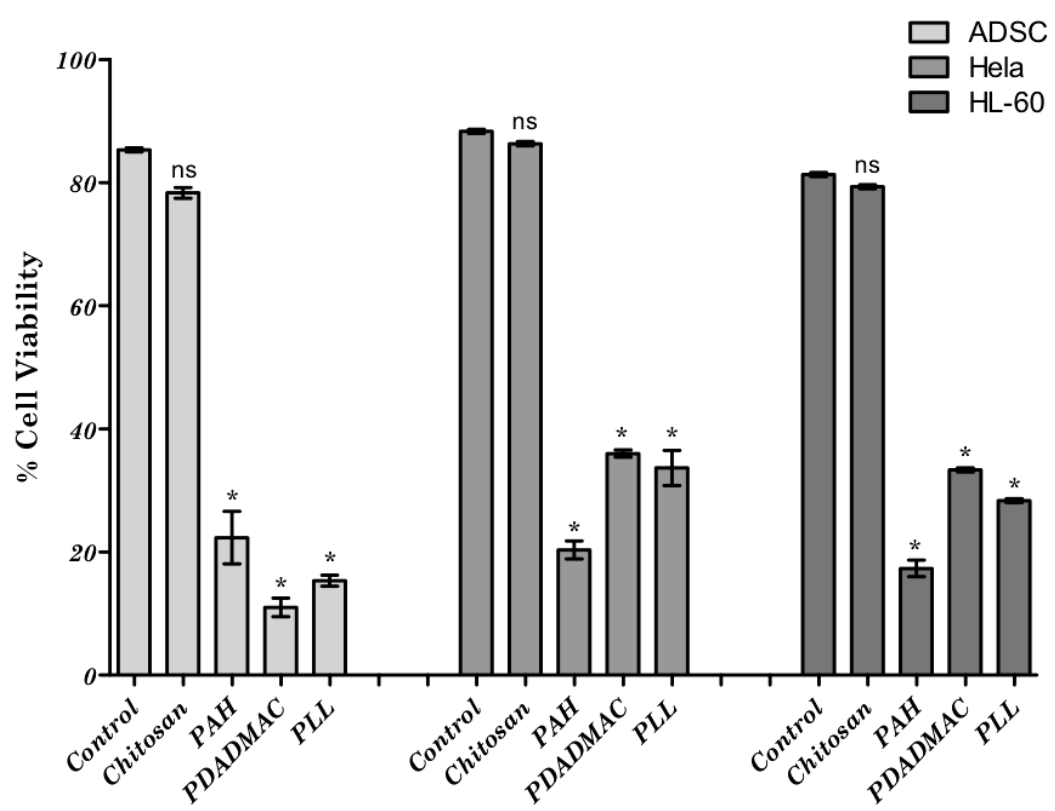

(A)
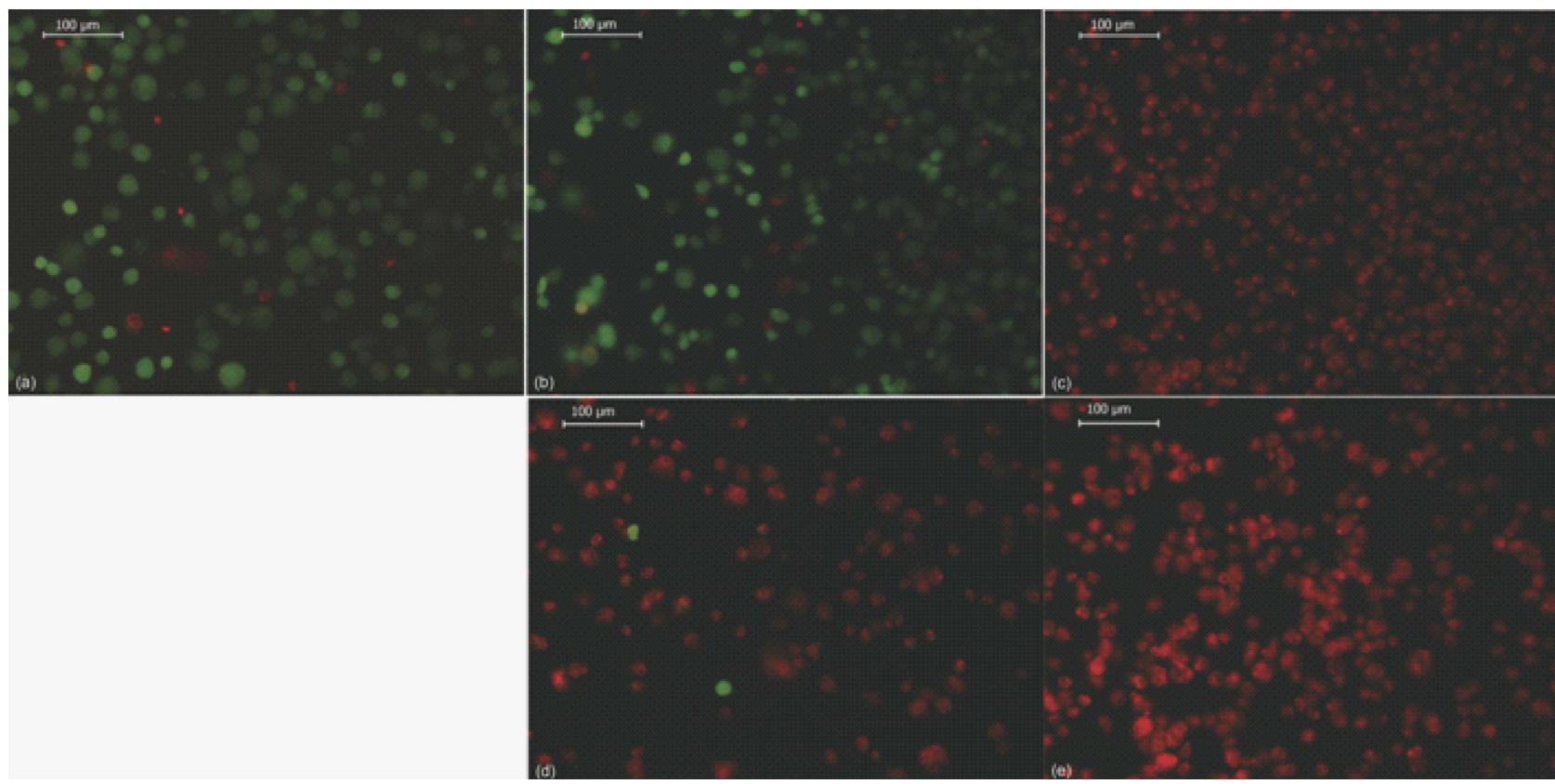

(B)

Figure 4. Effect of different polycations on cell viability after nanoencapsulation. (A) shows the effect of different polycation depositions on ADSC, Hela and HL-60 cell viability. In control cells, no polycations were added. The bars represent the mean of 3 experiments $+/-$ SEM. (*) p-value of less than 0.01 was statistically significant. (ns) = no significant. (See text for abbreviations); (B) shows cell viability in nanoencapsulated ADSCs assayed with different polycations: control (a), chitosan (b), PAH (c), PDADMAC (d) and PLL (e) by fluorescence microscopy. Living cells are shown in green (calcein staining) and dead cells in red (ethidiumhomodimer-1 staining). Magnification $200 \times$. The images are representative of at least 3 independent experiments.

Nevertheless, the preservation of an adequate membrane potential and its effects on cell viability remain to be elucidated.

We conclude that charge density is a key factor during nanocapsule formation. This observation implies that we must control the amount of polyelectrolytes deposited in each layer, which can manipulate other factors during this process, including $\mathrm{pH}$ and incubation time, when using non-excess concentrations of polyelectrolites and temperature. 
Studies performed by Germain et al. [17] on the encapsulation of MELN cells using Syto-16 as a marker of cell viability showed that PDADMAC exhibited the best index of cell viability compared to PEI (Poly(ethylene imine)), PPP (Poly(phosporic acid)), PS (protamine sulfate), PAH and PLL. Although these results appear to contradict our results, differences during the nanoencapsulation process should be considered between both studies. Germain's group used the MELN cell line (derived from MCF-7 breast cancer) and attached cells instead of cells in suspension. Thus, it is possible that the cell viability of the nanoencapsulation process varies according to the experimental conditions. First, a cancer-derived cell line is more resistant to stress conditions and exhibits reduced apoptosis [29]. Second, adherent cells are more stable in this way than in suspension. Finally, and perhaps most importantly, nanoencapsulation of adherent cells only coated on half of their cell surface. Therefore, these features allow cells to better resist layer depositions. As previously discussed, the method used by Germain and colleagues only coats half of the cell surface and is thus limited to applications where the cells are adhered to a scaffold. This may represent an advantage in our method because the cells in our study are completely coated on the cell surface, allowing them to be used in suspension prior to tissue engraftment.

Another issue to consider during nanocapsule formation is the nature of the polyelectrolytes used in the study. Natural polyelectrolytes are more biocompatible (particularly polysaccharides), innocuous and less toxic to cells than synthetic ones. Moreover, chitosan is a biocompatible natural polymer consisting of a unique polycationic polysaccharide. It is found in components of extracellular matrices and the exoskeleton of crustaceans, fungi and insects. It does not produce sensibilization and allergies when tested. The hydroxyl groups of its polysaccharide structure maintain a high state of hydration and keep away functional charged groups of the cell membrane at the moment of chitosan deposition while not affecting cell viability [18]. It is most likely that hyaluronic acid; chondroitin sulfate and other polyanions (i.e., alginate) are innocuous for this reason as well. On the other hand, poly-L-lysine is a semi-synthetic polyelectrolyte. It has been described as a biocompatible polymer and is commonly used in biomedical studies [30]. PAH and PDADMAC are synthetic polymers; however, their biocompatibility has not been fully described.

In addition, the concentration effect of chitosan during polycation deposition had no effect on cell viability (data not shown), and $0.5 \mathrm{mg} / \mathrm{mL}$ was used to avoid interferences from the non-dissolved chitosan.

Finally, as shown in Figure 5, the cell viability assays for the entire nanoencapsulation process showed that the percentage of living cells in each layer deposition re- mained at approximately $80 \%$ and was not statistically significant when compared to controls (non-encapsulated cells). Indeed, the cell viability after the first chitosan layer deposition was at $79 \%$, and after the second layer deposition of a mixture of hyaluronic acid and chondroitin sulfate was at $81 \%$ (Figure 5). The addition of extra layers did not alter cell viability (data not shown), suggesting a strong influence of the first polycationic layer deposition.

\subsection{Influence of Saline Solution on Cell Viability}

Germain et al. [17] described that the use of $147 \mathrm{mM}$ potassium-enriched saline solutions stabilized the cell membrane potential and that the presence of sodium had toxic effects on cells. We conducted experiments to evaluate what was the best saline solution to support cell viability under our conditions of nanoencapsulation. As shown in Figure 6(A), cell viability assays using different saline solutions demonstrated that both HBSS and Ringer's solution were effective in maintaining cell viability during the nanoencapsulation process, with viabilities ranging from $79 \%$ and $66 \%$, respectively. In our conditions, potassium-enriched saline solutions such as $\mathrm{HBSS} / \mathrm{KCl}$ and Ringer's $/ \mathrm{KCl}$ markedly decreased cell viability (36\% and $39 \%$, respectively). These results were consistent with the results obtained using the Live/ Dead assay kit (Figure 6(B)).

The appropriate choice of saline solution is useful not only to control nanoencapsulation but also to maintain

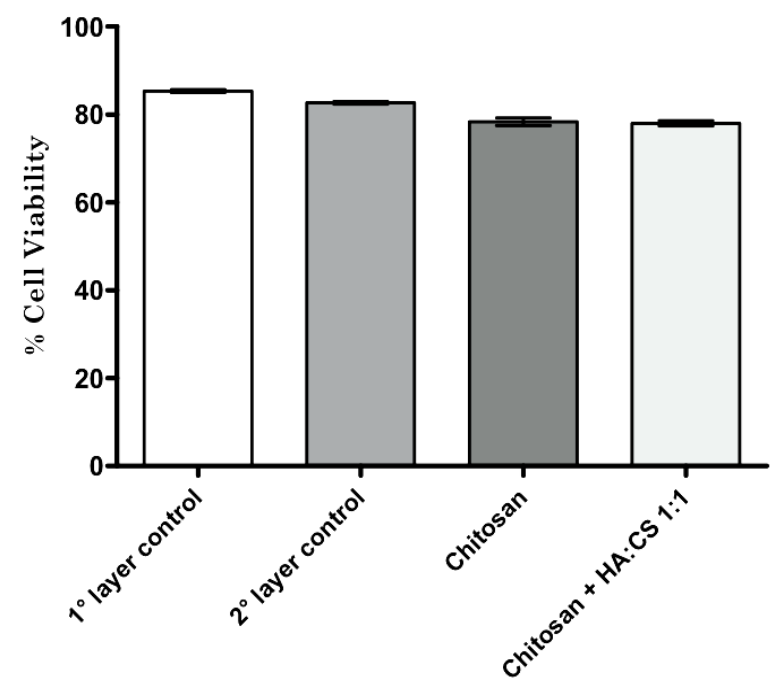

Figure 5. Cell viability after thenanoencapsulation procedure for ADSCs. The bar graph shows the cell viability of ADSCs after nanoencapsulation with chitosan as a first layer and a mixture of HA:CS 1:1 as the second layer. Controls were treated with the same process but in the absence of polyelectrolytes. The bars represent the mean of 3 independent experiments $+/-$ SEM. (*) p-value of less than 0.01 was statistically significant. $(\mathrm{ns})=$ no significant. 


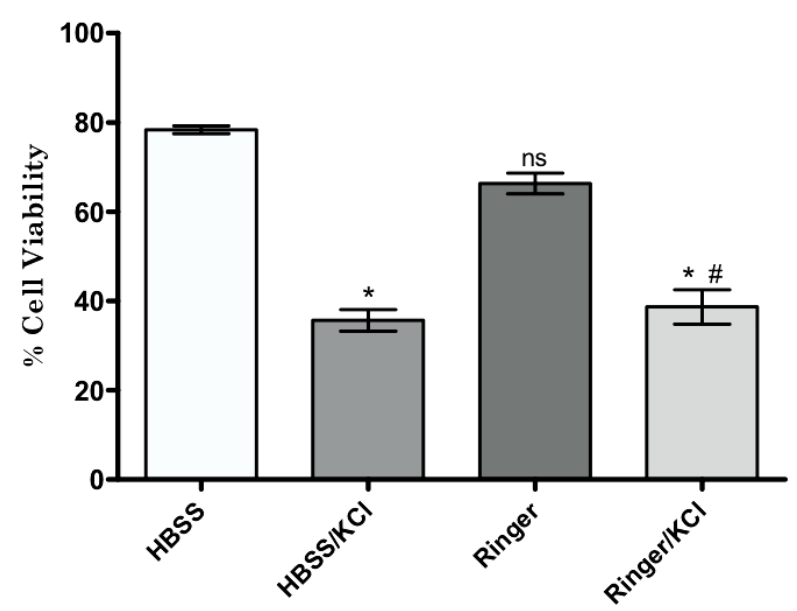

(A)

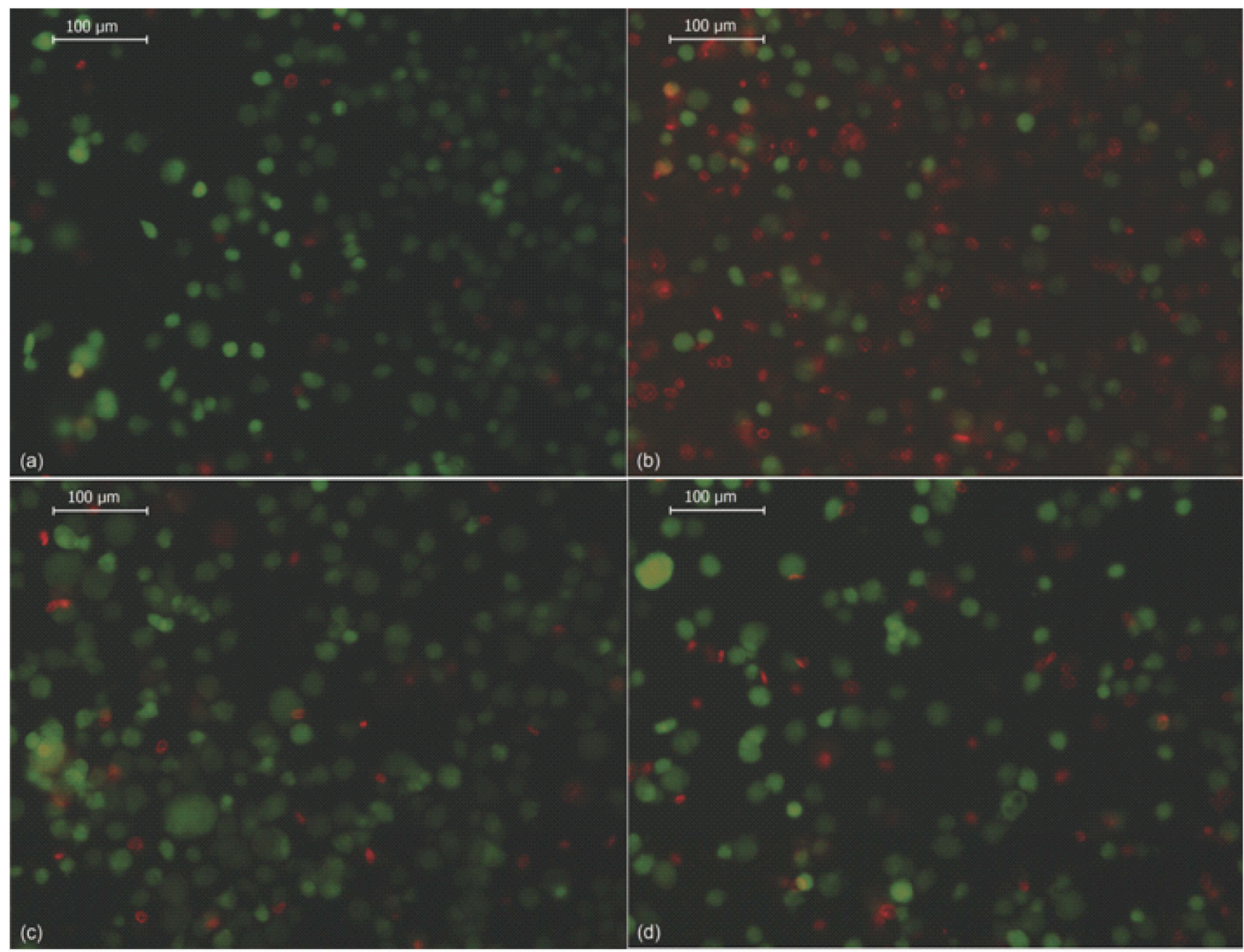

(B)

Figure 6. Effect of saline solution on cell viability after nanoencapsulation. (A) shows the effect of different saline solutions on ADSC cell viability after chitosan $(500 \mathrm{mg} / \mathrm{mL})$ deposition. The bars represent the mean of 3 experiments $+/-$ SEM. $(*)$ Statistically significant at $p<0.01$ with respect to control, (\#) statistically significant at $p<0.01$ with respect to Ringer's solution; (B) shows representative images of cell viability determination by fluorescence microscopy. Nanoencapsulated ADSCs were incubated as follows: HBSS (a), HBSS/KCl (b), Ringer's (c) and Ringer's/KCl (d). Living cells are shown in green (calcein staining) and dead cells are shown in red (ethidium homodimer-1 staining). Magnification $200 \times$. The images are representative of at least 3 independent experiments. 
cell viability. Saline solutions stabilize polyelectrolyte charges, regulating ionic strength and the $\mathrm{pH}$ of the solution, and maintain a compatible osmolarity for living cells. For this purpose, solutions must contain an adequate concentration of salts and other components that constitute buffers in the solution. It is fundamental to supplement media with proper sources of glucose for cell nutrition, as the time of nanoencapsulation, although not extensive, takes at least 30 minutes per layer and the total time of the procedure depends on the total number of layers to be deposited. In contrast to the results obtained by Germain et al. [17], we showed that $\mathrm{HBSS} / \mathrm{KCl}$, Ringer's/KCl (Figure 6(A)); both potassium-enriched saline solutions, resulted in a marked decrease in cell viability compared to standard and control solutions (HBSS and Ringer's). Thus, it is possible that the potassium requirements vary from different cell types not recommended during ADSC nanoencapsulation. Another requirement to be considered in the selection of saline solutions is the beneficial effects conferred by the presence of calcium and magnesium, both of which have an important role in the rapid re-sealing of damaged cell membranes [17].

\subsection{Functionality of Nanoencapsulated ADSC}

We established a methodology to evaluate functionality in ADSCs after nanoencapsulation. Although ADSCs were phenotypically characterized by the presence of surface antigens such as CD73, CD90 and CD105 and the absence of CD14, CD19, CD34, CD45 and HLA-DR [4] (data not shown), their functionality was evaluated through their ability to adhere to plastic dishes, proliferate and differentiate into adipocytes and osteoblasts [4]. These features require that nanocapsules maintain unaltered cell-to-cell interactions and cell-to-environment interactions. Consequently, nanoencapsulated ADSCs showed normal adhesion, growth and spreading on polyethylene dishes during a $24 \mathrm{~h}$ evaluation period and did not show differences compared to non-encapsulated cells.

The proliferation (growth) capacity is a central feature of MSCs. MSCs multiply without generating committed cell lineages. Using an MTT assay, our results showed that the growth rate is similar in both populations of cells (non-encapsulated vs. encapsulated), with a constant K equal to 0.2 and a doubling population time of 3 days with an approximate 10-fold increase at the end of the experiment (Figure 7). Cell proliferation involves multiple cell phenomena, beginning with cell adhesion, followed by mitosis and cell growth. As the growth rate remains unaltered after nanoencapsulation, all types of interactions in the cell and microenvironment are allowed, including cell division. There are several questions re- garding how cells divide after nanoencapsulation and whether the coating is randomly or equally distributed. Both aspects remain to be elucidated.

In addition, the differentiation capacity, an important feature on MSC [4], was also evaluated. Unmodified ADSCs (Figures 8(a)-(d)) and nanoencapsulated ADSCs (Figures 8(e)-(h)) were plated and evaluated for signs of differentiation into adi pocytes (21 days) and osteoblasts (28 days) after the proper addition of differentiation factors to the culture medium. As shown in Figures 8(f) and (h), nanoencapsulated ADSCs maintained their ability to differentiate into multiple mesenchymal cell lineages. Adipocytes were also easily identified by the presence of small red-colored fat droplets in the cytoplasm after specific staining with Red Oil O (Figures 8(b) and (f)). In addition, osteoblast differentiation could be detected under the microscope by the presence of an intense redcolored mineral deposit of calcium phosphate when the dye Alizarin Red S was added to the cells (Figures 8(d) and (h)).

\section{Conclusion}

Finally, we conclude that nanoencapsulation of ADSCs with chitosan as a first layer and a mixture of hyaluronic acid and chondroitin sulfate as a second layer successfully preserves the viability and functionality of human adipose-derived mesenchymal stem cells. On the other hand, the polycations PAH, PDADMAC and PLL dramatically decrease cell viability. In addition, the selection of saline solution can have mild effects on cell viability compared to the polycation effects; however, potassium-enriched saline solutions are not recommended for ADSC nanoencapsulation because of decreased cell viability. Moreover, nanoencapsulation does not interfere

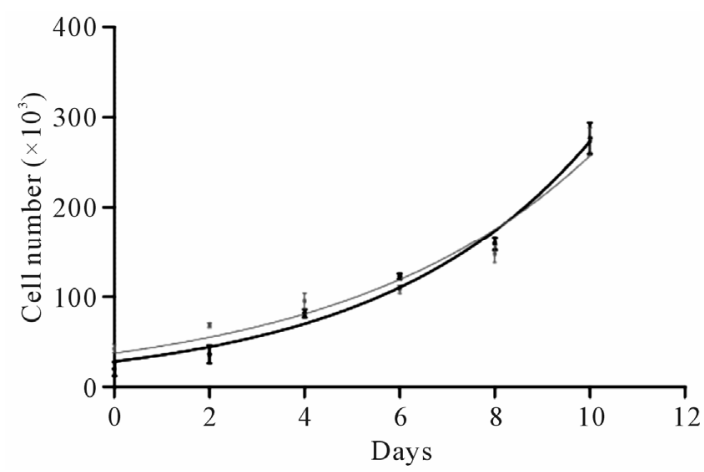

Figure 7. Effect of nanoencapsulation on ADSC proliferation. After ADSC nanoencapsulation (chitosan and a mixture of HA:CS 1:1, black curve), cell proliferation was evaluated for 10 days and compared to non-nanoencapsulated cells (gray curve). The proliferation curves showed no differences between the nanoencapsulated versus nonnanoencapsulated cells. Each point represents the mean of 3 independent experiments + / SEM. 


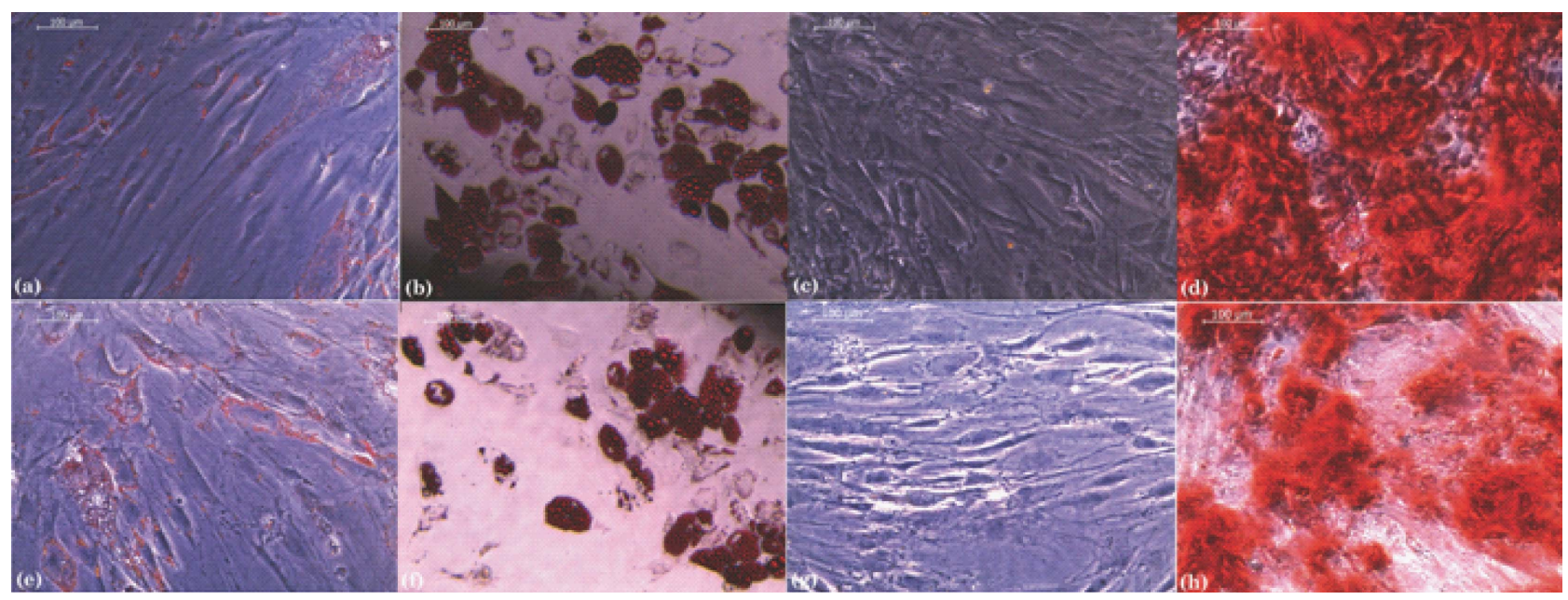

Figure 8. Effect of nanoencapsulation on ADSC differentiation into different cell lineages. Controls (a) to (d) and nanoencapsulated ADSCs (e) to (h) using chitosan and a mixture of HA:CS 1:1 as a second layer were stimulated to induce lineage differentiation. Adipocyte differentiation was evident through the accumulation of red lipid droplets in the cytoplasm as revealed by the Oil red O staining on both ADSCs (b) and nanoencapsulated ADSCs (f). Osteogenic differentiation was visualized by the accumulation of red calcium phosphate deposits by Alizarin red staining on both ADSC (d) and nanoencapsulated ADSC (h). (a), (c), (e) and (g) correspond to the respective unstimulated control cells. Magnification 200 $\times$. The images are representative of at least 3 independent experiments.

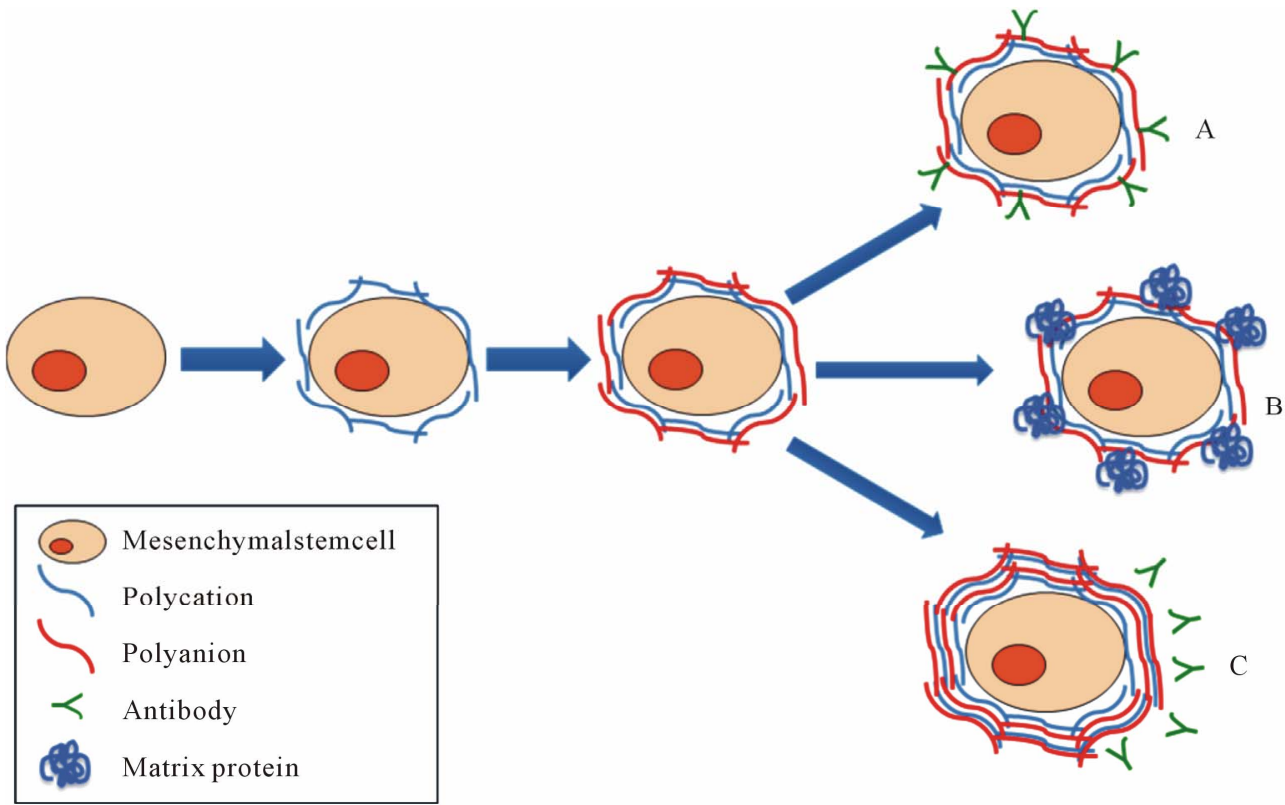

Figure 9. Layer-by-Layernanoencapsulation method and potential practical applications. Cell nanoencapsulation is based on the technique called Layer-by-Layer (LbL) deposition. LbL is formed by polymeric layers or film depositions that are mediated by alternating opposite electrostatic interactions on a charged template. Mesenchymal stem cells (MSC) may serve as a promising platform for cell-based encapsulation in regenerative medicine, both cellular therapy as well as tissue engineering. As indicated in the figure, nanoencapsulation of MSC could be useful for example for: (A) Cell targeting by conjugating a specific antibody; (B) Tissue engineering by incorporating a matrix protein that could be used as a scaffold; (C) Immunoisolation when using multilayer nanoencapsulation.

with the cell functionality of ADSCs, such as adhesiveness, proliferative activity and cell differentiation, which are phenomena that are highly dependent on cell-to-cell and cell-to-microenvironment interactions. Therefore, potential practical applications for nanoencapsulated
ADSCs, after the establishment of successful experimental conditions, include among others: cell targeting by conjugating a specific antibody; tissue engineering by incorporating a matrix protein that could be used as a scaffold; and immunoisolation when using multilayer 
nanoencapsulation (Figure 9).

\section{Acknowledgements}

This work was supported by the Fondo de Fomento al Desarrollo Científico y Tecnológico (FONDEF) grant D07i1014.

\section{REFERENCES}

[1] H. E. Young, C. Duplaa, R. Katz, T. Thompson, K. C. Hawkins, A. N. Boev, et al., "Adult-Derived Stem Cells and Their Potential for Use in Tissue Repair and Molecular Medicine," Journal of Cellular and Molecular Medicine, Vol. 9, No. 3, 2005, pp. 753-769. doi:10.1111/j.1582-4934.2005.tb00510.x

[2] K. Le Blanc, "Mesenchymal Stem Cells (MSCs) in Immunotherapy," Xenotransplantation, Vol. 14, 2007, pp. 426-427.

[3] K. Le Blanc and O. Ringden, "Immunomodulation by Mesenchymal Stem Cells and Clinical Experience," Journal of Internal Medicine, Vol. 262, No. 5, 2007, pp. 509-525. doi:10.1111/j.1365-2796.2007.01844.x

[4] M. Dominici, K. Le Blanc, I. Mueller, I. Slaper-Cortenbach, F. Marini, D. Krause, et al., "Minimal Criteria for Defining Multipotent Mesenchymal Stromal Cells," The International Society for Cellular Therapy Position Statement, Cytotherapy, Vol. 8, No. 4, 2006, pp. 315-317. doi:10.1080/14653240600855905

[5] A. Schaffler and C. Buchler, "Concise Review: Adipose Tissue-Derived Stromal Cells-Basic and Clinical Implications for Novel Cell-Based Therapies," Stem Cells, Vol. 25, No. 4, 2007, pp. 818-827. doi:10.1634/stemcells.2006-0589

[6] H. Mizuno, “Adipose-Derived Stem Cells for Tissue Repair and Regeneration: Ten Years of Research and a Literature Review," Journal of Nippon Medical School, Vol. 76, No. 2, 2009, pp. 56-66. doi:10.1272/jnms.76.56

[7] A. Diaspro, D. Silvano, S. Krol, O. Cavalleri and A. Gliozzi, "Single Living Cell Encapsulation in Nano-Organized Polyelectrolyte Shells," Langmuir, Vol. 18, No. 13, 2002, pp. 5047-5050. doi:10.1021/la025646e

[8] S. Krol, A. Diaspro, R. Magrassi, P. Ballario, B. Grimaldi, P. Filetici, et al., "Nanocapsules: Coating for Living Cells," IEEE Transactions on Nanobioscience, Vol. 3, No. 1, 2004, pp. 32-38. doi:10.1109/TNB.2004.824279

[9] S. Krol, S. del Guerra, M. Grupillo, A. Diaspro, A. Gliozzi and P. Marchetti, "Multilayer Nanoencapsulation. New Approach for Immune Protection of Human Pancreatic Islets," Nano Letters, Vol. 6, No. 9, 2006, pp. 19331939. doi:10.1021/n1061049r

[10] J. T. Wilson and E. L. Chaikof, "Challenges and Emerging Technologies in the Immunoisolation of Cells and Tissues," Advanced Drug Delivery Reviews, Vol. 60, No. 2, 2008, pp. 124-145. doi:10.1016/j.addr.2007.08.034

[11] H. Uludag, P. De Vos and P. A. Tresco, "Technology of Mammalian Cell Encapsulation," Advanced Drug Delivery Reviews, Vol. 42, No. 1-2, 2000, pp. 29-64.
doi:10.1016/S0169-409X(00)00053-3

[12] S. Krol, O. Cavalleri, P. Ramoino, A. Gliozzi and A. Diaspro, "Encapsulated Yeast Cells Inside Paramecium Primaurelia: A Model System for Protection Capability of Polyelectrolyte Shells," Journal of Microscopy, Vol. 212, No. 3, 2003, pp. 239-243. doi:10.1111/j.1365-2818.2003.01251.x

[13] S. Krol, M. Nolte, A.Diaspro, D. Mazza, R. Magrassi, A. Gliozzi, et al., "Encapsulated Living Cells on Microstructured Surfaces," Langmuir, Vol. 21, No. 2, 2005, pp. 705-709. doi:10.1021/la047715q

[14] A. Diaspro, "Nanocapsules: A European Community Interdisciplinary Network in the Nanobiosciences," IEEE Transactions on Nanobioscience, Vol. 3, No. 1, 2004, pp. 1-2. doi:10.1109/TNB.2004.824255

[15] Z. L. Zhi, B. Liu, P. M. Jones and J. C. Pickup, "Polysaccharide Multilayer Nanoencapsulation of InsulinProducing Beta-Cells Grown as Pseudoislets for Potential Cellular Delivery of Insulin," Biomacromolecules, Vol. 11, No. 3, 2010, pp. 610-616. doi:10.1021/bm901152k

[16] N. G. Veerabadran, P. L. Goli, S. S. Stewart-Clark, Y. M. Lvov and D. K. Mills, "Nanoencapsulation of Stem Cells within Polyelectrolyte Multilayer Shells," Macromolecular Bioscience, Vol. 7, No. 7, 2007, pp. 877-882. doi:10.1002/mabi.200700061

[17] M. Germain, P. Balaguer, J. C. Nicolas, F. Lopez, J. P. Esteve, G. B. Sukhorukov, et al., "Protection of Mammalian Cell Used in Biosensors by Coating with a Polyelectrolyte Shell," Biosensors and Bioelectronics, Vol. 21, No. 8, 2006, pp. 1566-1573. doi:10.1016/j.bios.2005.07.011

[18] P. K. Dutta, M. N. Ravikumar and J. Dutta, "Chitin and Chitosan for Versatile Applications," Journal of Macromolecular Science, Part C: Polymer Reviews, Vol. 42, No. 3, 2002, pp. 307-354. doi:10.1081/MC-120006451

[19] P. Angermann, "Glucosamine and Chondroitin Sulfate in the Treatment of Arthritis," Ugeskrift for Laeger, Vol. 165, No. 5, 2003, pp. 451-454.

[20] P. Hoffman, K. Meyer and A. Linker, "Transglycosylation during the Mixed Digestion of Hyaluronic Acid and Chondroitin Sulfate by Testicular Hyaluronidase," Journal of Biological Chemistry, Vol. 219, 1956, pp. 653-663.

[21] D. K. Singh and A. R. Ray, "Biomedical Applications of Chitin, Chitosan, and Their Derivatives," Journal of Macromolecular Science, Part C: Polymer Reviews, Vol. 40, No. 1, 2000, pp. 69-83.

[22] B. A. Bunnell, M. Flaat, C. Gagliardi, B. Patel and C. Ripoll, "Adipose-Derived Stem Cells: Isolation, Expansion and Differentiation," Methods, Vol. 45, No. 2, 2008, pp. 115-120. doi:10.1016/j.ymeth.2008.03.006

[23] N. V. Krishnamurthy and B. Gimi, "Encapsulated Cell Grafts to Treat Cellular Deficiencies and Dysfunction," Critical Reviews ${ }^{T M}$ in Biomedical Engineering, Vol. 39, No. 6, 2011, pp. 473-491.

doi:10.1615/CritRevBiomedEng.v39.i6.10

[24] A. Goren, N. Dahan, E. Goren, L. Baruch and M. Machluf, "Encapsulated Human Mesenchymal Stem Cells: A Unique Hypoimmunogenic Platform for Long-Term 
Cellular Therapy," The FASEB Journal, Vol. 24, No. 1, 2010, pp. 22-31. doi:10.1096/fj.09-131888

[25] T. Bhaiji, Z. Zhi and J. C. Pickup, "Improving Cellular Function and Immune Protection via Layer-by-Layer Nanocoating of Pancreatic Islet b-Cell Spheroids Cocultured with Mesenchymal Stem Cells," Journal of Biomedical Materials Research Part A, Vol. 100A, No. 6, 2012, pp. 1628-1636. doi:10.1002/jbm.a.34111

[26] A. Matsuzawa, M. Matsusaki and M. Akashi, "Effectiveness of Nanometer-Sized Extracellular Matrix Layer-by Layer Assembled Films for a Cell Membrane Coating Protecting Cells from Physical Stress," Langmuir, 2012 (online). doi:dx.doi.org/10.1021/la303459v

[27] A. H. Poghosyan, L. H. Arsenyan, H. H. Gharabekyan, J. Koetz and A. A. Shahinyan, "Molecular Dynamics Study of Poly(diallyldimethylammonium chloride) (PDADMAC)/Sodium Dodecyl Sulfate (SDS)/Decanol/Water
Systems," Journal of Physical Chemistry B, Vol. 113, No. 5, 2009, pp. 1303-1310. doi:10.1021/jp806289c

[28] J. M. Lourenco, P. A. Ribeiro, A. M. B. do Rego, F. M. Braz Fernandes, A. M. Moutinho and M. Raposo, "Counterions in Poly(allylamine hydrochloride) and Poly(styrene sulfonate) Layer-by-Layer Films," Langmuir, Vol. 20, No. 19, 2004, pp. 8103-8109. doi:10.1021/la049872v

[29] I. A. Cree, "Cancer Biology," Cancer Biology, Vol. 731, 2011, pp. 1-11. doi:10.1007/978-1-61779-080-5_1

[30] M. Chittchang, N. Salamat-Miller, H. H. Alur, D. G. Vander Velde, A. K. Mitra and T. P. Johnston, "POLY(Llysine) as a Model Drug Macromolecule with which to Investigate Secondary Structure and Microporous Membrane Transport, Part 2: Diffusion Studies," Journal of Pharmacy and Pharmacology, Vol. 54, No. 11, 2002, pp. 1497-1505. doi:10.1211/002235702108 